\title{
ROOSTER: a machine-learning analysis tool for Kepler stellar rotation periods
}

\author{
S. N. Breton ${ }^{1}$, A. R. G. Santos ${ }^{2}$, L. Bugnet ${ }^{1}$, S. Mathur ${ }^{3,4}$, R. A. García ${ }^{1}$, and P. L. Pallé 3,4 \\ 1 AIM, CEA, CNRS, Université Paris-Saclay, Université Paris Diderot, Sorbonne Paris Cité, 91191 Gif-sur-Yvette, France \\ e-mail: sylvain.breton@cea.fr \\ 2 Space Science Institute, 4765 Walnut Street, Suite B, Boulder, CO 80301, USA \\ ${ }^{3}$ Instituto de Astrofísica de Canarias, 38200 La Laguna, Tenerife, Spain \\ 4 Universidad de La Laguna, Dpto. de Astrofísica, 38205 La Laguna, Tenerife, Spain
}

Received 20 November 2020 / Accepted 25 January 2021

\begin{abstract}
In order to understand stellar evolution, it is crucial to efficiently determine stellar surface rotation periods. Indeed, while they are of great importance in stellar models, angular momentum transport processes inside stars are still poorly understood today. Surface rotation, which is linked to the age of the star, is one of the constraints needed to improve the way those processes are modelled. Statistics of the surface rotation periods for a large sample of stars of different spectral types are thus necessary. An efficient tool to automatically determine reliable rotation periods is needed when dealing with large samples of stellar photometric datasets. The objective of this work is to develop such a tool. For this purpose, machine learning classifiers constitute relevant bases to build our new methodology. Random forest learning abilities are exploited to automate the extraction of rotation periods in Kepler light curves. Rotation periods and complementary parameters are obtained via three different methods: a wavelet analysis, the autocorrelation function of the light curve, and the composite spectrum. We trained three different classifiers: one to detect if rotational modulations are present in the light curve, one to flag close binary or classical pulsators candidates that can bias our rotation period determination, and finally one classifier to provide the final rotation period. We tested our machine learning pipeline on 23431 stars of the Kepler $\mathrm{K}$ and $\mathrm{M}$ dwarf reference rotation catalogue for which $60 \%$ of the stars have been visually inspected. For the sample of 21707 stars where all the input parameters are provided to the algorithm, $94.2 \%$ of them are correctly classified (as rotating or not). Among the stars that have a rotation period in the reference catalogue, the machine learning provides a period that agrees within $10 \%$ of the reference value for $95.3 \%$ of the stars. Moreover, the yield of correct rotation periods is raised to $99.5 \%$ after visually inspecting $25.2 \%$ of the stars. Over the two main analysis steps, rotation classification and period selection, the pipeline yields a global agreement with the reference values of $92.1 \%$ and $96.9 \%$ before and after visual inspection. Random forest classifiers are efficient tools to determine reliable rotation periods in large samples of stars. The methodology presented here could be easily adapted to extract surface rotation periods for stars with different spectral types or observed by other instruments such as K2, TESS or by PLATO in the near future.
\end{abstract}

Key words. methods: data analysis - stars: solar-type - stars: activity - stars: rotation - starspots

\section{Introduction}

Low-mass stars with convective outer layers (hereafter solartype stars) can exhibit magnetic activity (e.g., Brun \& Browning 2017). In the Sun, one of the manifestations of magnetic activity is the emergence of magnetic spots at its surface. While for stars other than the Sun, one cannot directly image starspots in great detail, one can in principle detect their impact on, for example, stellar brightness. As the star rotates, starspots come in and out of the visible hemisphere, thus modulating the stellar brightness. As a consequence, such spot modulation encloses information on stellar magnetic activity and surface rotation (e.g., Berdyugina 2005; Strassmeier 2009).

Surface rotation periods represent a key parameter to understand stellar angular momentum transport. This process is not yet sufficiently understood in order to be correctly implemented in stellar evolution codes (e.g., Aerts et al. 2019, and references therein). Neglecting angular momentum transport may have a significant impact on the stellar age estimates (e.g., Eggenberger et al. 2010). These stellar ages are crucial for studying the evolution of the Milky Way (e.g., Miglio et al. 2013), as well as for a better characterisation of planetary systems (e.g., Huber 2018). The evolution of planetary systems, which is driven by tidal and magnetic effects, is indeed strongly influenced by the stellar rotation rate, through angular momen- tum exchange between planets and their host star star (e.g., Zhang \& Penev 2014; Mathis 2015; Bolmont \& Mathis 2016; Strugarek et al. 2017; Benbakoura et al. 2019).

The surface rotation period is found to be a strong function of the stellar age: low-mass stars with an external convective envelope spin down during their main-sequence evolution. The first empirical relation between the two stellar parameters was proposed by Skumanich (1972, also known as Skumanich spin-down law). In particular, for young main-sequence solartype stars, the rotation period can be used to constrain stellar ages through the so-called gyrochronology relations (e.g., Barnes 2003, 2007; Mamajek \& Hillenbrand 2008; Meibom et al. 2011, 2015; García et al. 2014a). However, for stars older than the Sun, the ages determined by gyrochronology deviate from the asteroseismic ages (Angus et al. 2015; van Saders et al. 2016). Angus et al. (2020) also show that the empirical gyrochronology relations cannot reproduce the stellar ages inferred from velocity dispersion. These discrepancies justify the need for improved gyrochronology relations. Furthermore, the presence of surface rotational modulation in the light curves has a limiting effect on the detection of low-amplitude transiting exoplanets (e.g., Cameron 2017). The amplitude of those rotational modulations is directly linked to the surface stellar magnetic activity level and leads to difficulties in performing asteroseismic analysis of active solar-like stars because this magnetism inhibits pulsations 
(e.g., García \& Ballot 2019; Mathur et al. 2019, and references therein).

During its four-year nominal mission, the Kepler satellite (Borucki et al. 2010) collected high-quality, long-term, and nearly continuous photometric data for almost 200000 targets (Mathur et al. 2017) in the Cygnus-Lyra region. After the failure of two reaction wheels, Kepler was reborn as a new mission called K2 (Howell et al. 2014). K2 observed more than 300000 targets around the ecliptic (Huber et al. 2016) over 20 three-month campaigns. The Transiting Exoplanet Survey Satellite (TESS, Ricker et al. 2015) observed nearly the all sky during its nominal mission. TESS gathered data for tens of millions of stars with an observational length ranging from 27 days to one year. This large amount of observations represent the best photometric dataset to study the distribution of stellar surface rotation periods, $P_{\text {rot }}$. But to reach this goal and extract periods for large samples of stars, automatic procedures are required. Such an attempt was recently undertaken with a deep learning approach using convolutional neural networks (Blancato et al. 2020). However, training those networks requires a heavy computational power. Hence, the development of an easy-to-train machine learning procedure coupled with the outputs of widelyused rotation pipelines will be an extremely valuable asset. Indeed, this is the objective of the current study.

Random forest (RF) algorithms (Breiman 2001) are either able to classify large samples of stars (when used for classification) or to provide parameter estimates (when used for regression). In asteroseismology, RF algorithms have already been used to estimate stellar surface gravities ( $\log g$, Bugnet et al. 2018) and to automatically recognise solar-like pulsators (Bugnet et al. 2019). They have also recently been used to perform analyses linked to surface stellar rotation, but only in order to infer long rotation periods from TESS 27-day-long light curves (Lu et al. 2020). In this work, we present the Random fOrest Over STEllar Rotation (ROOSTER), which is designed to select a rotation period for stars observed by Kepler through a combination of RF classifiers applied to a variety of methods used to extract $P_{\text {rot }}$ and different ways to correct the light curves. Thus, the main goal of ROOSTER is to automatically achieve a large degree of reliability that could even be improved by performing a small number of human visual validation of certain results, which are also suggested by the pipeline.

The layout of the paper is as follows. Section 2 presents the sample of $\mathrm{K}$ and $\mathrm{M}$ stars used in this work as well as the methodology to extract the surface rotation periods. Section 3 describes all the parameters used to feed ROOSTER. In Sect. 4, we explain how the ROOSTER pipeline is built and we detail the training scheme we chose for this work. In Sect. 5, we present the rotation periods obtained with the ROOSTER pipeline and the strategy implemented for stars with missing input parameters. Results, limitations, and possibilities to increase the accuracy of ROOSTER are discussed in Sect. 6. Finally, conclusions and perspectives are given in Sect. 7 .

\section{Observations and methodology to extract surface rotation periods}

The target sample of this work is comprised of main-sequence $\mathrm{K}$ and $\mathrm{M}$ stars observed by the Kepler mission. This sample was the one analysed in the rotation study done by Santos et al. (2019). It is the most complete rotation catalogue for the Kepler $\mathrm{K}$ and $\mathrm{M}$ dwarfs. Hereafter, this catalogue is referred as S19. In it, 26521 targets were selected according to the Kepler Stellar Properties Catalogue for Data Release 25 (DR25, Mathur et al.
2017) and for 15290 of them, a $P_{\text {rot }}$ was provided. As the purpose of this work is rotation analysis, different types of potential contaminants are removed from our study, namely Kepler objects of interest, eclipsing binaries, misclassified red giants, misclassified RR Lyrae, light curves with photometric pollution by nearby stars or multiple signals, classical pulsators (namely $\delta$ Scuti and/or $\gamma$ Doradus) candidates, and contact binary candidates (see S19 for details on the contaminants).

S19 determined average rotation periods, $P_{\text {rot }}$, and average photometric activity indexes ( $S_{\mathrm{ph}}$, Mathur et al. 2014), for more than $60 \%$ of the targets. In fact, about $30 \%$ of the reported $P_{\text {rot }}$ by S19 are new detections in comparison with the previous largest $P_{\text {rot }}$ catalogue (McQuillan et al. 2014). In addition to an automatic selection of reliable $P_{\text {rot }}$, the S19 analysis required an extensive amount of visual examinations (see Sect. 2.2), which justifies the need for a pipeline such as ROOSTER.

\subsection{Light curve preparation}

We use three sets of KEPSEISMIC light curves $^{1}$, the same used in S19. KEPSEISMIC data is calibrated using KADACS (Kepler Asteroseimic Data Analysis and Calibration Software; García et al. 2011), which applies customised apertures and corrects for outliers, jumps, drifts, and discontinuities at the edges of the Kepler Quarters. Because regular interruptions in the observations (such as the gaps induced every three days due to the angular momentum dump of the Kepler satellite) could produce a false detection of $P_{\text {rot }}$, gaps shorter than 20 days are filled by implementing inpainting techniques using a multi-scale discrete cosine transform (García et al. 2014b; Pires et al. 2015).

We use in particular three different data sets obtained with three high-pass filters, with cutoff periods at 20, 55, and 80 days. The transfer function is unity for periods shorter than the cutoff period, while for longer periods it varies sinusoidaly, slowly approaching zero at twice the cutoff period. Thus, for a given filter, it is possible to recover periods longer than the respective cutoff period. The main goal of using and comparing three different filters is to find the best trade-off between filtering the undesired instrumental modulations at long periods while keeping the intrinsic stellar rotational signal. KEPSEISMIC light curves are optimised for asteroseismic studies, but are also very well suited for rotation and magnetic activity studies (see for example Appendix B in S19).

\subsection{Methodology to extract $P_{\text {rot }}$}

Surface rotation periods can be studied with different methods, namely: periodogram analysis (e.g., Reinhold et al. 2013; Nielsen et al. 2013); Gaussian processes (e.g., Angus et al. 2018); gradient power spectrum analysis (e.g., Shapiro et al. 2020; Amazo-Gómez et al. 2020); autocorrelation function (ACF, e.g., McQuillan et al. 2013, 2014); time-period analysis based on wavelets (e.g., Mathur et al. 2010; García et al. 2014a); or with a combination of different diagnostics such as the composite spectrum (CS, e.g., Ceillier et al. 2016, 2017; Santos et al. 2019). Using artificial data, Aigrain et al. (2015) compared the performance of different pipelines used to determine the rotation. The authors found that the combination of the data set preparation and different rotation diagnostics, in particular the one implemented in this work (see next paragraph), yields the best set of results in terms of reliability and completeness.

\footnotetext{
1 KEPSEISMIC data are available at MAST via http://dx.doi. org/10.17909/t9-mrpw-gc07
} 
In this work, we use the procedure followed in S19 to extract $P_{\text {rot }}$ combining three analysis methods (time-period analysis, $\mathrm{ACF}$, and CS) with the KEPSEISMIC light curves. Those $P_{\text {rot }}$ are respectively denoted $P_{\mathrm{ACF}}, P_{\mathrm{GWPS}}$ and $P_{\mathrm{CS}}$. Control parameters related to the $\mathrm{ACF}$ and $\mathrm{CS}$ are also extracted, they are denoted $H_{\mathrm{ACF}}, G_{\mathrm{ACF}}$, and $H_{\mathrm{CS}}$. Each method also allows us to compute a magnetic activity proxy $S_{\mathrm{ph}}$. Description and details concerning those parameters can be found in Appendix A.

During the visual examination in S19, a group of classical pulsators (CP) and close binaries (CB) candidates was identified. Those stars are referred hereafter as Type $1 \mathrm{CP} / \mathrm{CB}$ candidates. These targets are typically fast rotators $\left(P_{\text {rot }}<7\right.$ days $)$, showing very stable and fast beating patterns, a large number of harmonics in the power spectrum, and/or very large $S_{\mathrm{ph}}$ values. Interestingly, Simonian et al. (2019) found that the majority of the fast rotators observed by Kepler are tidally-synchronised binaries. In fact, there is a significant overlap between the Type $1 \mathrm{CP} / \mathrm{CB}$ candidates and the tidally-synchronised binaries (see S19). This suggests that the modulation seen in the light curve of such objects may still be related to rotation but not of single targets. Therefore, we advice caution while studying such targets. In $\mathrm{S} 19$, the Type $1 \mathrm{CP} / \mathrm{CB}$ candidates were flagged through visual inspection.

\section{Parameters used to feed the machine learning algorithms}

To ensure that ROOSTER is able to identify targets with detectable rotation modulation and to extract reliable rotation periods, the selection of relevant parameters is crucial. They will help the algorithm to perform successive classifications as described in Sect. 4. Indeed, a random forest algorithm relies on the following principle: during the training step, a forest of classification trees is grown to perform splits between the elements with different labels (Breiman 2001). From the root of the tree, the training sample is progressively separated in purer (considering the labels) sub-samples. This task is performed by selecting at each iteration the best split over a range of randomly generated range of splits using the input parameters.

The three rotation periods $\left(P_{\mathrm{ACF}}, P_{\mathrm{GWPS}}\right.$, and $\left.P_{\mathrm{CS}}\right)$ obtained thanks to the method described in Sect. 2.2 and in Appendix A, combined with the three sets of data corresponding to the three different high-pass filtered KEPSEISMIC light curves (20, 55 and 80 days), yield a total of 9 rotation-period candidates for each star. The objective is to train the algorithm to choose the rotation period given in S19. To do so, we also feed our pipeline with the parameters (amplitude, central value and standard deviation) of the five first Gaussian functions fitted on the GWPS and the CS. If, for a given star, the $i$ th Gaussian function could not be fitted, the corresponding parameters are set to zero. We also include the $\chi^{2}$ of the two fits, the number of Gaussian functions fitted to each GWPS and CS and the mean level of noise. This makes 108 additional input parameters (36 for each filter). As only a few stars have a sixth Gaussian function fitted for both GWPS and CS method, we verified that keeping the corresponding parameters out of the classification does not affect the result. We also feed the random forest algorithm with the $H_{\mathrm{ACF}}, G_{\mathrm{ACF}}$, and $H_{\mathrm{CS}}$ for each filter, and the $S_{\mathrm{ph}}$ with corresponding errors for each method and each filter, leading to 27 more parameters.

Because the rotation periods depend on the global parameters of the stars (age, spectral type, etc.), we complement our set of input parameters with the effective temperature, $T_{\text {eff }}$, and logarithm of surface gravity $\log g$ from the DR25 catalogue (Mathur et al. 2017). We also decide to use the Flicker in Power metric (FliPer, Bugnet et al. 2018), which is correlated with the stellar surface gravity. FliPer is a measure of the total power in the stellar power spectral density (PSD) between a low frequency cut-off, $v_{\mathrm{C}}$ and the Nyquist frequency, $v_{\mathrm{N}}$. It can be defined as:

FliPer $\left(v_{\mathrm{C}}\right)=\frac{1}{v_{\mathrm{N}}-v_{\mathrm{C}}} \int_{v_{\mathrm{C}}}^{v_{\mathrm{N}}} \operatorname{PSD}(v) \mathrm{d} v-P_{n}$

where $P_{n}$ is an estimate of the photon noise level which depends on the magnitude of the star. In this work, we used a noise estimation calibrated with the whole set of KEPSEISMIC calibrated light curves. We consider the following four FliPer values: $F_{0.7}$, $F_{7}, F_{20}, F_{50}$ for $v_{\mathrm{C}}$ of $0.7,7,20$ and $50 \mu \mathrm{Hz}$, respectively.

Additional parameters linked to the quality of the stellar targets and of the acquired light curves are added: Kepler magnitude values $K_{p}$ from the Kepler input catalogue (Brown et al. 2011), length of the light curves (in days), bad quarter flag, number of bad quarters in the light curve, and finally, the start time and end time of the light curve.

For each star, a total of 159 input parameters are used to feed the ROOSTER pipeline. The full list of parameters is given in Table B.1.

\section{The ROOSTER pipeline}

ROOSTER uses three random forest classifiers ${ }^{2}$ : RotClass, which selects stars that have a rotation signal; PollFlag, which flags the Type-1 CP/CB candidates; and PeriodSel, which allows ROOSTER to choose the final rotation period between the three different filters and the three analysis methods. Due to its ability to take into account a large number of parameters, its flexibility, and its adaptability to the training set, the random forest algorithm constitutes a clear improvement from the former method consisting in defining thresholds on a small number of key parameters.

The three classifiers are trained with the same 159 input parameters per star as defined in the previous section, but each classifier considers different training sets and gives different output labels. Depending on the task the classifier has to achieve, each of them may give different importance to the input parameters. The flow diagram of ROOSTER is shown in Fig. 1.

\subsection{Description}

The first ROOSTER classifier, RotClass, separates stars with rotational modulations (labelled Rot stars) from other stars (labelled NoRot stars). These NoRot stars are rejected and are no longer considered in the rest of the analysis (although some of those stars may later still be flagged for visual checks). An example of $R o t$ star, KIC 1164583, is presented in Fig. 2. In this figure, the light curves and the output of the three different rotation period retrieval methods (WPS/GWPS, ACF, and CS) are shown.

In general, the risk of confusion by the ROOSTER pipeline between a Rot and a NoRot star is a consequence of two main issues: it can be difficult to reliably detect low-amplitude rotational modulations and Kepler instrumental effects may introduce additional periodic modulations in the light curve.

A small fraction of Rot stars (generally stars with $P_{\text {rot }}<7$ days) may be close-in binaries or classical pulsator stars candidates. As mentioned above, the detected signal for this type of targets may not be consistent with rotation of single stars. The PollFlag classifier is trained to identify these CP/CP candidates and flag them as FlagPoll, while the rest of the stars are

2 The random forests classifiers are implemented with the Python scikit-learn package (Pedregosa et al. 2011). 


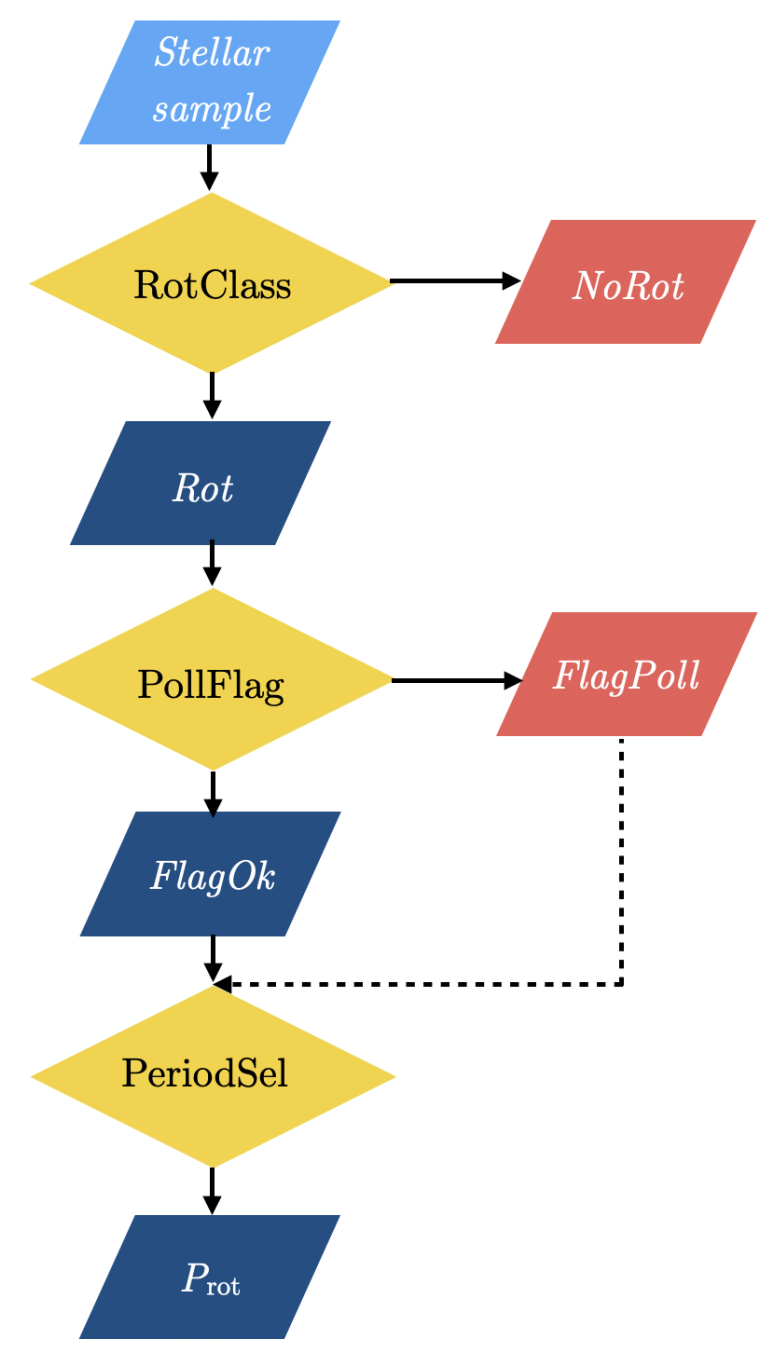

Fig. 1. Structure of the ROOSTER pipeline. The yellow diamonds outline the action of each classifier used by the machine learning algorithm. The stars of the sample (lightblue parallelogram) are anlaysed by the pipeline. The red and dark blue parallelograms emphasis the label attributed by the ROOSTER classifiers to each star of the sample. First, stars with rotational modulation are selected by RotClass and the label Rot or NoRot is given. NoRot stars are not considered in the subsequent analysis. The PollFlag classifier flags Type $1 \mathrm{CP} / \mathrm{CB}$ candidates (labelled FlagPoll). The remainder of the stars receive the FlagOk label. Finally, the PeriodSel classifier selects the rotation period.

labelled FlagOk. The light curve and PSD of the FlagPoll candidate, KIC 2283703, is shown as an example in Fig. 3. Its Type $1 \mathrm{CP} / \mathrm{CB}$ character can be recognised due to the large amplitude of the brightness variations, the beating pattern, and the large number of high-amplitude peaks in the PSD.

Finally, the third classifier, PeriodSel, is trained with the same 159 parameters defined in Sect. 2. This time, the labels of the training set correspond to the nine possible rotation periods $\left(P_{\mathrm{ACF}}, P_{\mathrm{CS}}, P_{\mathrm{GWPS}}\right.$ in the 20,55 , and 80 -day filters). The goal is to select the most reliable period among those nine estimates, which may differ. For example, Fig. 2 illustrates the impact of the high-pass filter when producing the light curves of KIC 1164583. The 55- and 80-day filters indicate a 47-day period, while the period detected from the 20-day filter is 21 days. The comparison between the three WPS shows that the 47-day signal has been filtered out by the 20-day filter. The WPS shows power at longer periods which is another evidence for a longer period being filtered. The 47-day GWPS period of the 55day filter is finally chosen as the retrieved $P_{\text {rot }}$. PeriodSel selects one of the nine estimates as the retrieved rotation period, $P_{\text {rot, ML }}$. We emphasise that the transfer function of a given filter slowly approaches zero at twice the cutoff period, that is to say it is possible to retrieve rotation periods longer than the cutoff period of the filter applied to the light curve.

\subsection{Training}

The followed approach allows us to show the robustness of ROOSTER. In particular, ROOSTER must be weakly dependent to the exact composition of the training set. This can be only achieved if the training-set parameter space distribution is similar to the test-set parameter space distribution. Hence, we decide to perform a training-loop process where the three classifiers are trained one hundred times. Each time, the full working sample is randomly divided in a training set (containing $75 \%$ of the stars) and a test set (containing $25 \%$ of the stars). The number of times each star is drawn in the test set over the one hundred training repetitions follows a binomial law with probability $p=0.25$ and number of trials $n=100$.

In total, the target sample of this study includes 23431 targets from S19: 21707 of them have all the parameters from the rotation pipeline (referred as the RotClass sample), while 1,724 have missing parameters (referred as the MissingParam sample, mainly due to missing ACF values). S19 gives measured $P_{\text {rot }}$ for 14936 stars: 14562 for the RotClass sample and 374 stars for the MissingParam sample. These stars are labelled Rot and are then used with the PeriodSel classifier (called the PeriodSel sample). A total of 8495 stars do not have a reliable $P_{\text {rot }}$ in S19. Hence, this S19 sample is ideal to train and evaluate the performance of ROOSTER. The MissingParam sample is not considered at this stage but will be used later in Sect. 5.2. The working PollFlag sample is constituted of 630 stars: 315 Type $1 \mathrm{CP} / \mathrm{CB}$ candidates completed at each training with 315 other stars randomly chosen from the PeriodSel sample. The composition of the different samples is summarised in Table 1.

An RF classifier not only gives a label to each classified star, its forest structure also allows obtaining the proportion of trees attributing a given class to each considered star. Indeed, the final classification of the target corresponds to the label that is selected by the majority of the trees. We can then define the classification ratio as the number of trees giving the output label over the total number of trees of the forest. This ratio is useful to estimate the reliability of the machine-learning output for each star.

Since we perform a training loop with random repetitions, we are also able to compute the mean classification ratio for each star. This mean classification ratio is computed as the mean of all the classification ratios found each time a star has been drawn in the test set.

\section{Results}

In what follows, the accuracy term is defined as the agreement of any label given by a ROOSTER classifier and the S19 catalogue taken as a reference.

From the 21707 stars of the RotClass sample, the mean classification ratio gives the right label (Rot or NoRot) for 20443 stars, which yields an overall accuracy of $\sim 94.2 \%$. We find that 1707 stars have been misclassified at least once during the 100 runs $(7.9 \%)$ while 1259 stars have been misclassified in half of the repetitions of the training they were part of the test set $(5.8 \%)$. Finally, 884 stars have been systematically misclassified 


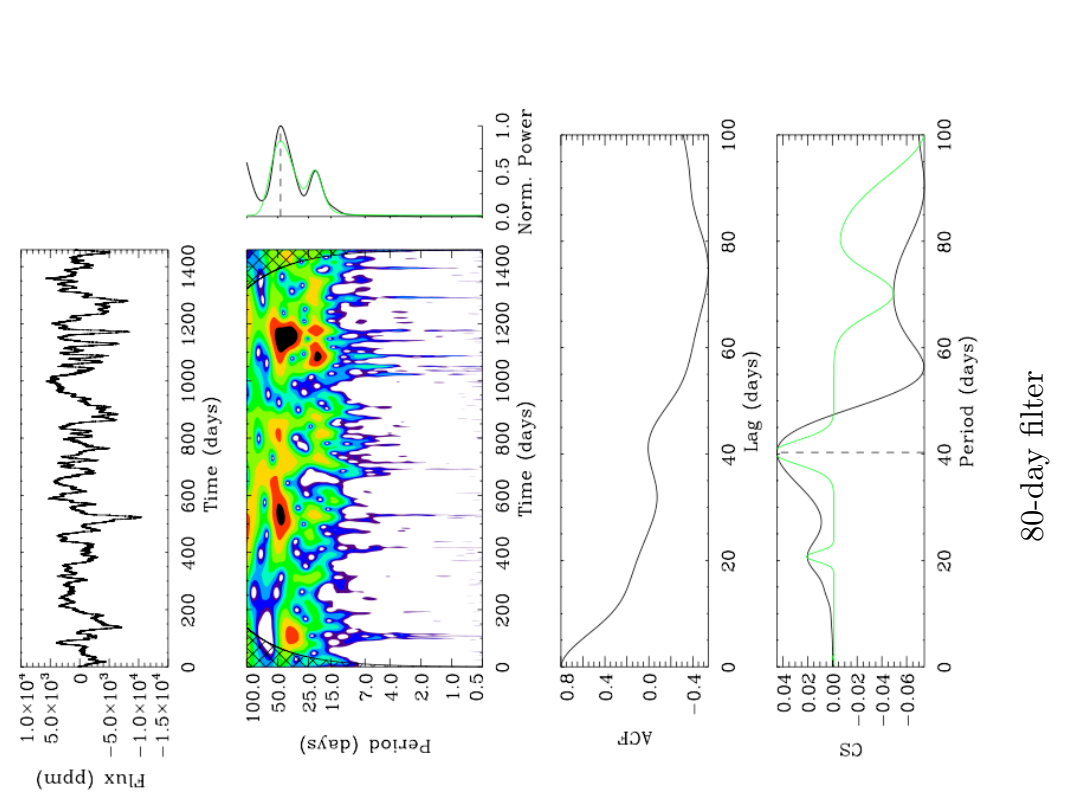

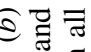

迸

范

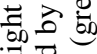

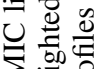

$\sum_{3}$

핑

II

过

둔

종 을

글를

茨苛

它

讨

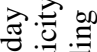

$\varnothing .0$

छ

in

กิ๊ 클

के
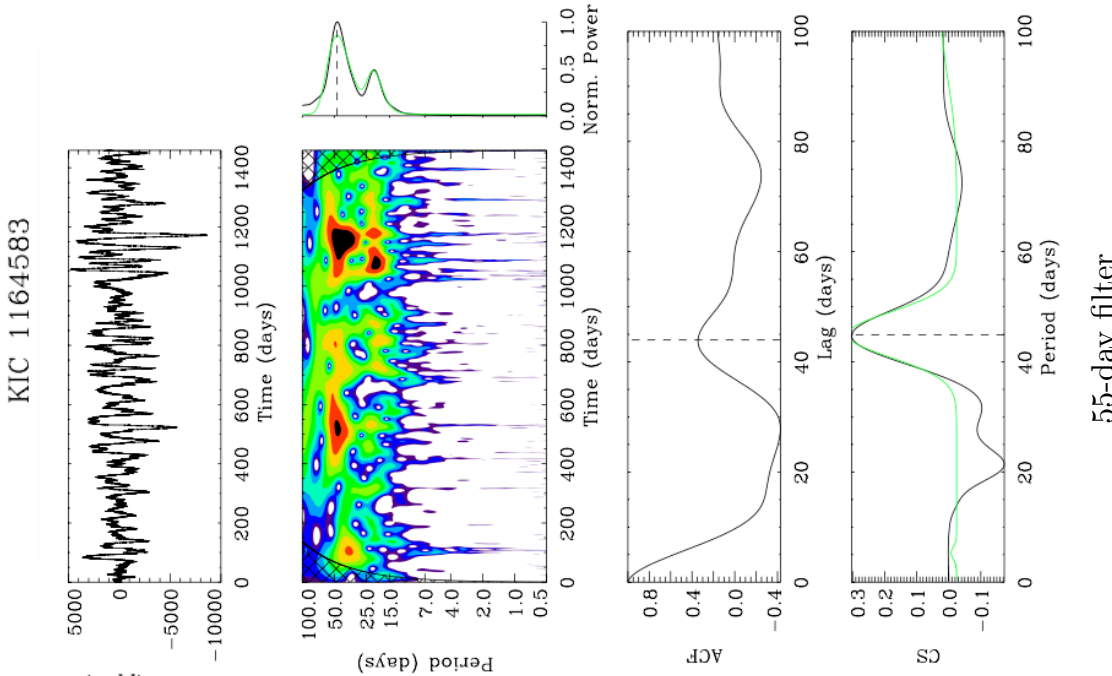

0 \&

s

今.

50 क

n

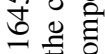

已

ปี

훈 홍

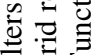

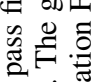

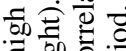

(udd) $x_{\text {IId }}$

(sKep) poị.rad

s.

.
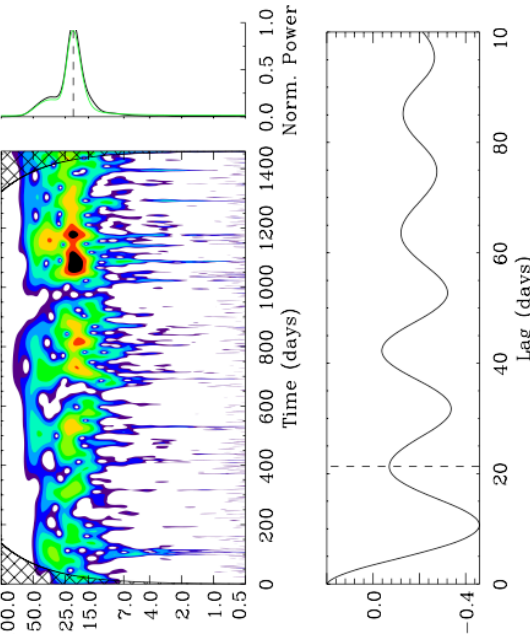

킬을.

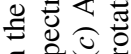

दी की

उٓ

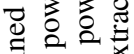

可 훵

$\circ \stackrel{\Xi}{0}$

ڤ

音零.

릉으으음

일음

๘

둴

ญ 巳

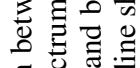

ठํㅠ

䒕

छิ

i 过 $\frac{0}{3}$

里紫范 

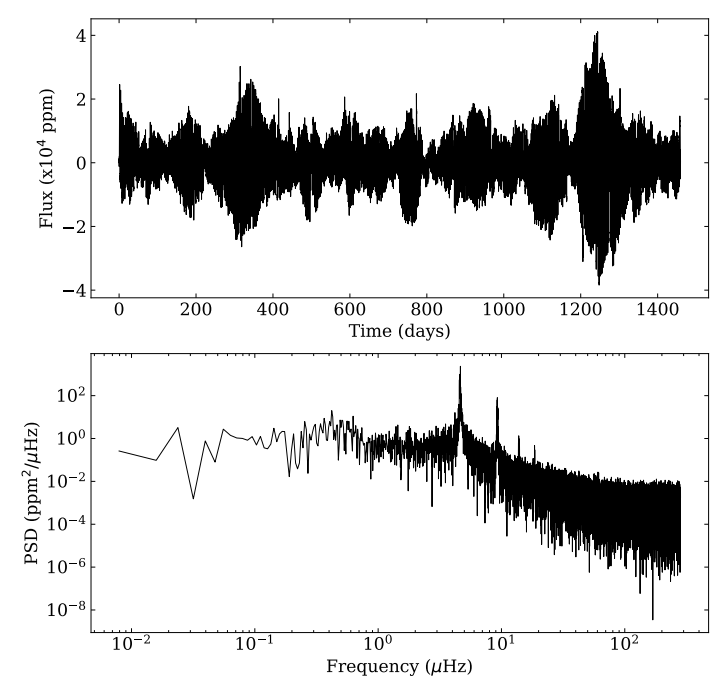

Fig. 3. PSD of the Type $1 \mathrm{CP} / \mathrm{CB}$ candidate KIC 2283703. The light curve is characterised by high-amplitude flux variation and beating patterns over time, while the PSD exhibits a large number of highamplitude peaks.

(4.1\%). The accuracy of RotClass is already valuable but can be improved with a limited amount of visual checks. By performing visual inspections, we verify that the highest number of misclassifications occur within the mean classification ratio between 0.4 and 0.8 . For this reason, stars within these mean classification ratios should be part of the visual verification for future target samples. In the case of the target sample of the current study, 2230 stars $(10.3 \%)$ are within this interval. One of the reasons for the misclassifications is the presence of instrumental modulations that can be mistaken by rotation periods.

Concerning PollFlag, the goal is to obtain a high sensitivity to real Type-1 CP/CB candidates, while keeping the fraction of misclassifications low. The sensitivity is here defined as the fraction of Type $1 \mathrm{CP} / \mathrm{CB}$ candidates correctly identified by PollFlag. Thus, the sensitivity does not take into account the fraction of non-Type $1 \mathrm{CP} / \mathrm{CB}$ candidates correctly identified. In this case, it is a more relevant estimator than the accuracy, as the fraction of Type-1 $\mathrm{CP} / \mathrm{CB}$ candidates in the sample is small. During the training loop, approximately $2.8 \%$ of the considered non-Type-1 $\mathrm{CP} / \mathrm{CB}$ candidates have been misclassified by PollFlag as Type-1 CP/CB candidates. If PollFlag had been applied blindly on the full PeriodSel sample, this means that approximately 400 non-Type- $1 \mathrm{CP} / \mathrm{CB}$ candidates would have been wrongly flagged. However, considering the mean classification ratio, only two Type-1 CP/CB candidates have been misclassified, which yields a PollFlag sensitivity of $\sim 99.4 \%$. Looking at the detailed results during the training loop, 6 Type-1 CP/CB candidates are misclassified at least once $(1.9 \%), 4$ are misclassified in half of the repetitions $(1.3 \%)$, and none is systematically misclassified.

Finally, the mean classification ratio of the PeriodSel classifier gives us an accuracy of $\sim 86.8 \%$, that is to say the label corresponding to the rotation period from S19 is attributed to 12638 stars over 14562 in the PeriodSel. Nevertheless, a significant fraction of the labels chosen by PeriodSel contains a $P_{\text {rot }}$ which is within $10 \%$ of the one in S19. For this reason, we adopt the agreement within $10 \%$ as the true accuracy, TA, of PeriodSel in the following way:

$\mathrm{TA}=\frac{\operatorname{Card}\left(E_{\text {good }}\right)}{\operatorname{Card}(E)} \times 100$, with $E$ being the considered ensemble of stars and $E_{\text {good }}$ the subsample from $E$ where PeriodSel retrieves a $P_{\text {rot,ML matching }}$ $P_{\text {rot,S19 }}$ within $10 \%$.

We find that PeriodSel selected an incorrect period for 830 stars at least once $(5.7 \%)$, for $693(4.8 \%)$ in half of the realisations and systematically for $569(3.9 \%)$. In terms of the mean classification ratio, a correct rotation period $P_{\text {rot,ML }}$ is retrieved for 13871 stars. Hence, the true accuracy is $\sim 95.3 \%$. Figure 4 shows the comparison between $P_{\text {rot,ML }}$ and the reference values from S19. From the 1924 stars for which the label attributed by ROOSTER was not the same as in S19, 1233 are within the shaded blue area of the figure. For the remaining $4.7 \%$ of the stars, we observe three cases:

1. Among the periods retrieved by ROOSTER, 103 of them lie within a tolerance threshold that is slightly over $10 \%$ (see the shaded green area of Fig. 4). This could be due to a precision difference between $P_{\mathrm{GWPS}}, P_{\mathrm{ACF}}$, and $P_{\mathrm{CS}}$ or to the filter that was selected to choose the value of the rotation period.

2. The machine learning mistakes instrumental modulations for real rotation signals when such signatures appear in the light curves. These instrumental modulations usually have periods between 38 and 50 days. 99 ROOSTER rotation periods lie both in this [38-50] days regions and outside of the $10 \%$ agreement region.

3. Depending on the rotation signature, some of the nine period estimates correspond to the second harmonic of the correct rotation period. PeriodSel privileges this harmonic over the fundamental period for 381 stars of the PeriodSel sample.

\subsection{Weight of the considered parameters}

Here we discuss briefly the importance given by the three classifiers to the input parameters. For each classifier, Fig. 5 presents the weights of the ten most important parameters. The rotation periods provided by the ACF and GWPS for the 55-day filter are in the top five parameters of RotClass. PollFlag favours the ACF parameters, which is coherent as the ACF shows a specific pattern for $\mathrm{CP} / \mathrm{CB}$ candidates. We note that PollFlag is the only classifier among the three that also prioritises errors on $S_{\mathrm{ph}}$ determination to perform the classification. For PeriodSel, three of the most important parameters are the rotation period values computed from the 55-day filtered light curves. The parameters of the Gaussian functions fitted to the GWPS and CS also have a strong influence over the classification. The remainder of the most important parameters are the $H_{\mathrm{ACF}}, G_{\mathrm{ACF}}$, and $H_{\mathrm{CS}}$ from the 20-day filtered light curves, which highlights the importance of those control parameters when considering the reliability of a potential rotation signal.

Finally, one could be concerned by the effect of systematic errors on $T_{\text {eff }}$ and $\log g$ parameters. However, because of the small weight of these two parameters, their influence on our classifiers' accuracy is marginal. Therefore, the impact on our methodology of any systematic error in both parameters is negligible. A similar conclusion will be reached in Sect. 6.2 where we tested our algorithm using simulated data by Aigrain et al. (2015).

\subsection{Strategy for stars with missing parameters}

The rotation pipeline does not always provide a value for all the parameters needed for the random forest classification. The majority of the missing parameters are related to the ACF for the 55- and 80-day filters. In general this happens because there is no clear peak in the ACF. However, to perform the classification, an 
Table 1. Composition of the different subsamples used for the study and scores of the different classifiers.

\begin{tabular}{lccccc}
\hline \hline & RotClass sample & PollFlag sample & \multicolumn{2}{c}{ PeriodSel sample } & MissingParam sample \\
\hline Number of input stars & 21707 & 315 Type 1 CP/CB candidates + 315 non-candidates & 14562 & 1724 \\
\hline RotClass accuracy (\%) & $94.2 / 97.6$ & - & - & 77.4 \\
PollFlag sensitivity (\%) & - & - & - & $50^{(*)}$ \\
PeriodSel accuracy (\%) & - & - & $95.3 / 99.5$ & 79.4 \\
Global accuracy (\%) & - & $92.1 / 96.9$ & - \\
\hline
\end{tabular}

Notes. The 315 non-candidate stars used in the PollFlag sample are randomly chosen in the PeriodSel sample at each training. For RotClass and PeriodSel, the metrics chosen to express the score is the accuracy while it is the sensitivity for PollFlag. When two scores are given, the first and the second one corresponds to the score before and after visual inspection, respectively. ${ }^{(*)}$ There is only 4 Type- $1 \mathrm{CP} / \mathrm{CB}$ candidates in this sample, among which, only 2 have been correctly classified yielding 50\% probability.

input value must be provided for each of the 159 parameters. To test ROOSTER on the stars with missing parameters, we choose to train our three classifiers without the values for $P_{\mathrm{ACF}}, S_{\mathrm{ph}, \mathrm{ACF}}$ and the respective uncertainty for the three filters. However, we decide to keep $H_{\mathrm{ACF}}$ and $G_{\mathrm{ACF}}$ as input parameters. If for a given star in a given filter, $P_{\mathrm{ACF}}$ is not determined, $H_{\mathrm{ACF}}$ and $G_{\mathrm{ACF}}$ will be set to zero. This is logical in the sense that the value for $G_{\mathrm{ACF}}$ and $H_{\mathrm{ACF}}$ indicates whether the corresponding peak is significant.

Like before, RotClass is trained with the RotClass sample. Only 1334 of the stars of the MissingParam sample are correctly classified (accuracy of $77.4 \%$ compared to the accuracy of $94.2 \%$ reached with the RotClass sample). This is not a surprise in the sense that when the ACF does not yield a period estimate, it suggests that the modulation in the light curve may not be clear enough. This assumption is confirmed when comparing the fraction of stars with rotation signal in the RotClass sample and in the MissingParam sample: $67.1 \%$ of the stars in the RotClass sample are labelled Rot in S19 while only $24 \%$ of the MissingParam sample are labelled Rot in S19. Thus, the two distributions are very different and this impacts the outcomes of the classifier.

The accuracy loss is similar for PeriodSel, with only $79.4 \%$ of rotation period retrieved within 10\%. Among the MissingParam sample, there are only four Type $1 \mathrm{CP} / \mathrm{CP}$ candidates, which make it difficult to estimate the sensitivity for this sample. Two of those candidates are correctly flagged $(50 \%)$.

In order to optimise the efficiency of the pipeline, it is thus preferable to be able to compute upstream a full set of parameters for a maximal number of stars and to avoid having to deal with a too large MissingParam sample.

\section{Discussion}

The RF classification with ROOSTER represents a clear improvement compared to the automatic selection method based on control parameters performed in S19 (see Appendix A). However, for a small fraction of the sample, the ROOSTER results differ from those in S19. Nevertheless, the discrepancies represent well-known issues that we try to flag.

Once we have obtained the rotation periods from the ROOSTER pipeline, we define a list of criteria so that we can flag stars that require visual checks and hence improve the accuracy of the final retrieval of extracted rotation periods.

1. First, we identify stars for which the proper filter is not selected. We expect $P_{\text {rot,ML }}$ chosen by PeriodSel to be coherent with the filter from which they were extracted. This means that, for $P_{\text {rot,ML }}<23$ days, $P_{\text {rot,ML }}$ should have been extracted from the 20-day filtered light curve, for 23 days $<P_{\text {rot,ML }}<60$ days

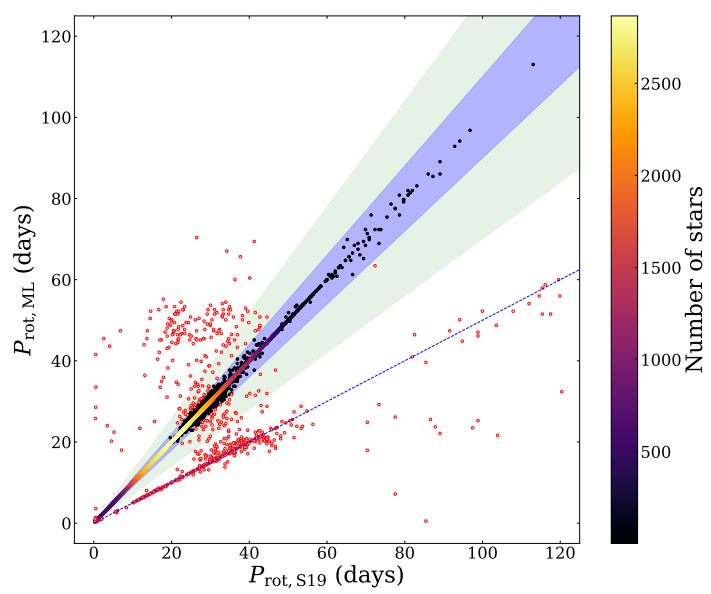

Fig. 4. Comparison between $P_{\text {rot,ML }}$ and the values given in $\mathrm{S} 19$ for the stars of the PeriodSel sample. The shaded blue and green areas highlight the 10 and $30 \%$ agreement regions, respectively. The number of stars inside this area is colour coded. The stars outside the shaded area are represented by red dots. The dashed blue line corresponds 2:1 line.

from the 55-day filtered light curve, for $P_{\text {rot,ML }}>60$ days from the 80-day filtered light curve. If $P_{\text {rot,ML agrees within } 15 \% \text { with }}$ the $P_{\mathrm{GWPS}}$ of the proper filter, we replace $P_{\text {rot,ML }}$ by this value. Otherwise, the star is flagged for visual check. This change is motivated by the goal of retrieving the most accurate $S_{\mathrm{ph}}$, which is generally obtained considering a light curve with a high-pass filter coherent with $P_{\text {rot,ML }}$.

2. As shown in Fig. 4, a significant number of stars lie on the 1:2 line, highlighting an issue with the harmonics of $P_{\text {rot }}$, which can partially be overcome. We flag targets for visual inspection if at least one of the other $P_{\text {rot }}$ estimates (nine in total) is within $15 \%$ of the double of $P_{\text {rot,ML }}$. We notice that this harmonics issue is generally linked to $P_{\text {rot }}$ yielded by the GWPS fitting method that selects a higher-order harmonic instead of the first one as it is more often done by the ACF methodology. In order to reduce the impact of this problem, it would be necessary to perform a comprehensive study of the fitted GWPS harmonic's pattern. This analysis is out of the scope of the current paper but will be the issue of a future upgrade of the ROOSTER pipeline.

3. The ROOSTER pipeline might also attribute the wrong rotation period because of instrumental modulation in Kepler data. The 55 and 80 day filtered light curves are the most affected by instrumental modulations. For these cases, $P_{\text {rot,ML }}$ is usually between 38 and 50 days, but can be longer. Those instrumental modulations can be identified through visual inspection of the light curves and of the control figures of the rotation code. For this reason, we flag all stars with $P_{\text {rot,ML }}>38$ days. 

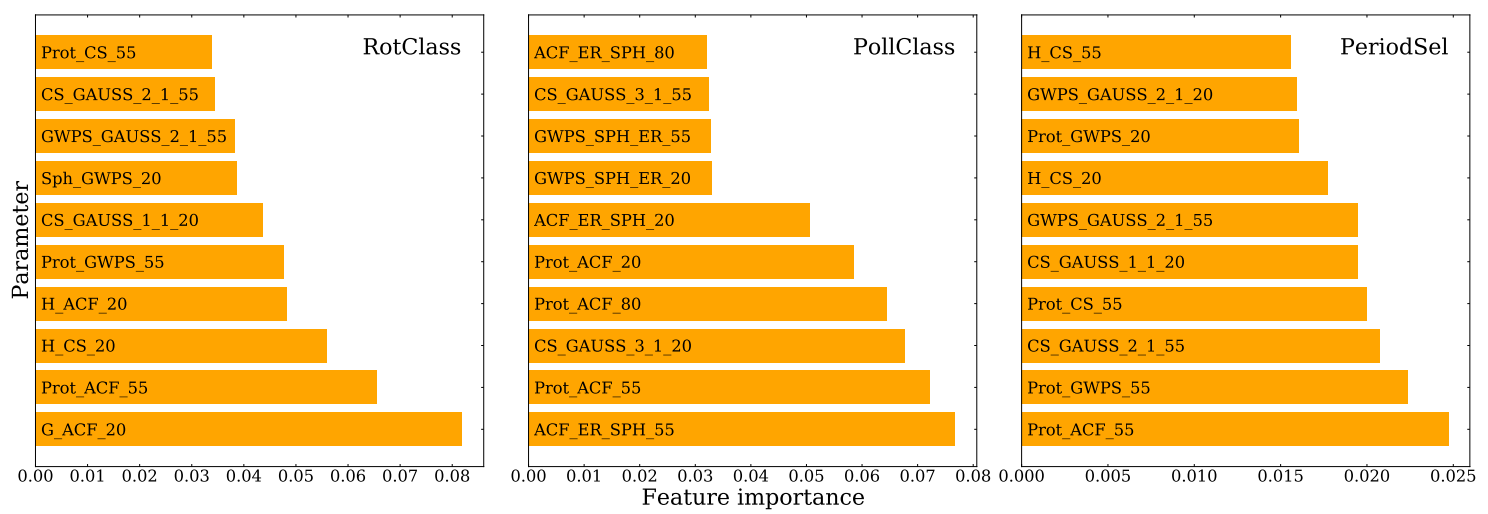

Fig. 5. Importance of the ten most important parameters for the three classifiers of the pipeline. The full list of ROOSTER input parameters is given in Appendix B.

Instrumental modulations can correspond to, for example: sudden bursts in flux; abnormal flux variations which only happen every four Kepler Quarters, that is to say every Kepler year; and flux variations that are present throughout the light curve with a main periodicity of one Kepler year, while in the rotation diagnostics used here one can identify its harmonics and the resulting filtered signals. We note that the 80-day filtered KEPSEISMIC light curves are naturally the most affected by instrumental modulations. However, the signature in the 20-day and 55-day filtered light curves may also have an instrumental origin. This is also true for other data products in the literature. Avoiding instrumental modulations is one of our objectives and one of the reasons to use three KEPSEISMIC light curves (Sect. 2.1) and perform complementary visual checks (see also discussion in S19).

4. Stars with $P_{\text {rot,ML }}<1.6$ days are also flagged to ensure that the detected signal is not $\mathrm{CP} / \mathrm{CB}$-like. Observationally, synchronised binaries correspond to targets with detected period shorter than seven days (Simonian et al. 2019).

5. All the Type $1 \mathrm{CP} / \mathrm{CB}$ candidates in $\mathrm{S} 19$ have a period shorter than seven days. We therefore flag for visual inspection Type-1 CP/CB candidates detected by PollFlag when $P_{\text {rot,ML }}>7$ days.

6. Stars for which the length of data or the presence of gaps might not be enough to cover the range of rotation periods expected for the studied stars are also visually inspected. The flagging criterion can be based on the total length of observations (given in days or quarters) and on the continuity of the light curve. In this work we have used a criterion in quarters. Stars were flagged if their light curve was shorter than five quarters.

7. The last group of visual checks correspond to stars are those for which the mean classification ration of RotClass is between 0.4 and 0.8 , as already stated in Sect. 5 .

The different types of stars flagged for visual inspection are summarised in Fig. 6.

The six conditions listed above result in flagging for visual inspection 4606 stars (31.6\% of the PeriodSel sample). Among those 4606 stars, 628 will be corrected after the visual inspection. Finally, for 27 stars, we prefer the value given by the machine learning over the S19 value. After this step, we obtain a $99.5 \%$ accuracy for the retrieval of $P_{\text {rot,ML }}$. It should be noted that among the 63 remaining stars outside the $10 \%$ agreement between ROOSTER and S19, 24 of them lie in a large tolerance zone of $30 \%$ of the good rotation period. As mentioned in Sect. 5, stars with mean classification ratio between 0.4 and 0.8 are visually checked. That means that some of the stars classified
NoRot by RotClass are also visually checked. The total number of visual checks to perform in the scheme we just described is then 5,461 (25.2\% of the RotClass sample), which represent a significant improvement compared to S19 (60\% of the sample was visually checked).

We are able to compute a global accuracy score for both RotClass and PeriodSel classifiers. Among the 14562 stars of the PeriodSel sample, both RotClass and PeriodSel made correct predictions for 13,413 stars $(92.1 \%$ of the sample), that is to say RotClass correctly labelled them Rot and $P_{\text {rot,ML agrees within }}$ $10 \%$ of the $P_{\text {rot }}$ given in S19. After the visual checks, this accuracy is raised to $96.9 \%$. The accuracy scores before and after visual inspection are summarised in Table 1.

We note that it could be possible to reduce the global amount of visual checks. For example, 683 stars are flagged only because their light curve was shorter than five quarters. It represents $4.7 \%$ of the PeriodSel sample. Among those 683 stars, only 7 of them are actually corrected after the visual inspection. The visual check of short light curves could therefore be avoided without a significant loss of accuracy. However, concerning the methodology described in this paper, we decided to clearly point out every risk of confusion that could occur in ROOSTER.

\subsection{Period retrieval in short light curves}

The ROOSTER accuracy on short light curves may yield interesting hints about the performance of our method on a dataset constituted by stars observed by TESS or K2. Our dataset contains 29 light curves with length within 30 and 35 days and 690 light curves with length within 35 and 150 days. PeriodSel accuracy over those two subsamples is $96.5 \%$ and $92.9 \%$, respectively. The $P_{\text {rot }}$ distribution for those two subsamples is presented in Fig. 7. All the stars with light curves length below 35 days present rotation periods below five days. The ability of our method to retrieve longer $P_{\text {rot }}$ in such short light curves is beyond the scope of this paper and will be discussed in details in a subsequent study.

\subsection{Period retrieval with simulated data}

ROOSTER was also applied on the simulated data of the hare and hounds exercise in Aigrain et al. (2015). The results of this analysis are presented in Appendix C. For the purpose of this exercise, ROOSTER was trained considering only light curves from S19 and had no previous knowledge of the simulated data. We found that ROOSTER applied blindly performed better than any of the methods compared in Aigrain et al. (2015). The 


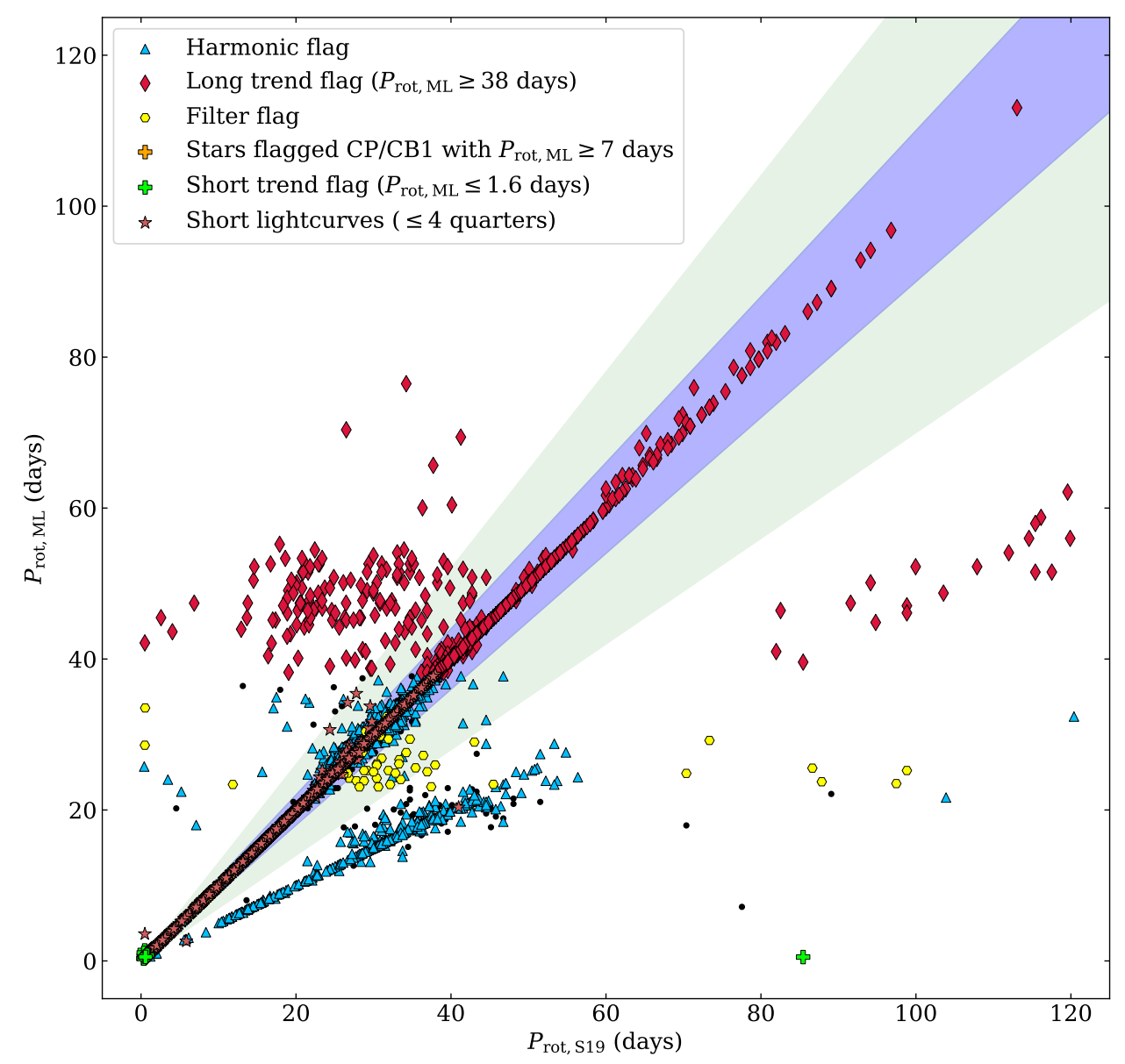

Fig. 6. Comparison between the ML retrieved period and the value given in S19. The $10 \%$ tolerance region is represented in blue, while the large tolerance zone (30\%) is shown in green. Stars that are flagged for visual checks are highlighted: yellow circle show the stars selected with the filter condition, red diamonds those by the long trend condition, cyan triangle those selected by harmonic search condition, brown stars those selected with the short light curve condition, green cross those selected by the short trend condition, and orange cross the stars flagged as Type $1 \mathrm{CP} / \mathrm{CB}$ with $P_{\text {rot,ML }}>7$ days.
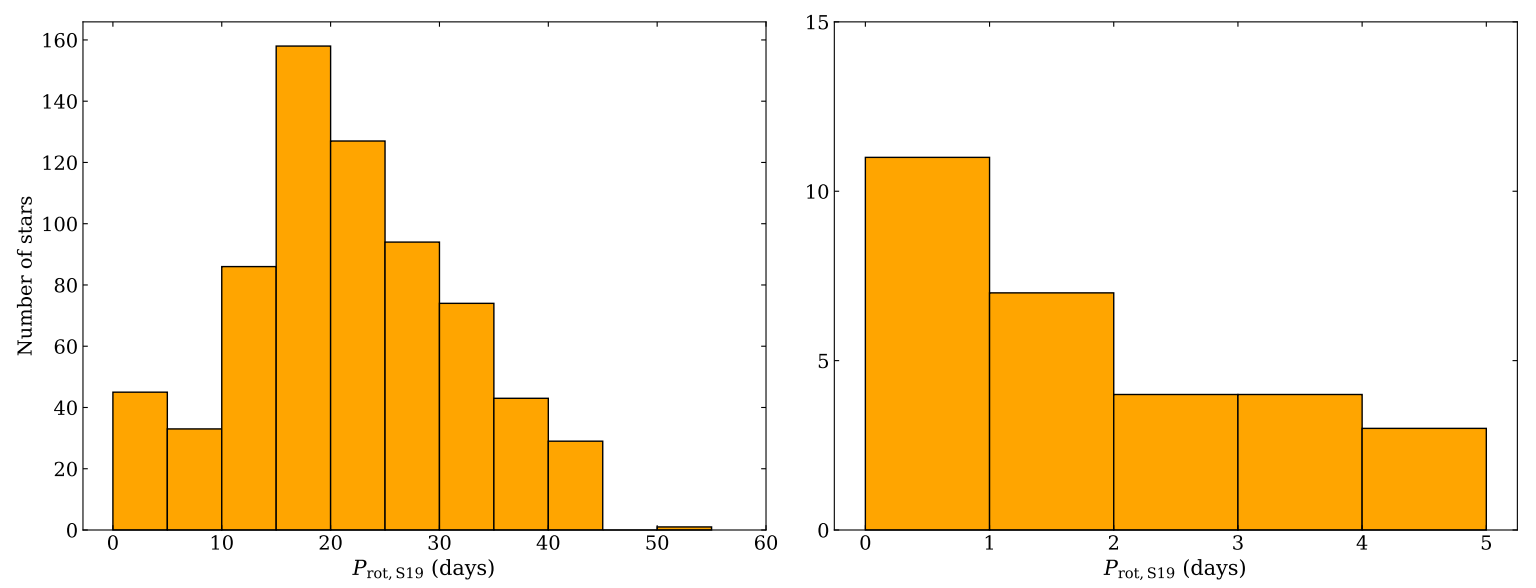

Fig. 7. Left: $P_{\text {rot }}$ distribution for stars with light curves within 35 and 150 days. Right: same as left panel, but for stars with light curves within 30 and 35 days.

ROOSTER accuracy scores are close to what we obtain on real data in this study. It is important to notice that in this analysis $T_{\text {eff }}$ and $\log g$ were not used in the training in order to have the same homogeneous set of parameters for the noise-free and noisy light curves.

\section{Conclusion}

In this paper, we have described ROOSTER, a machine learning analysis pipeline dedicated to obtain rotation periods with a small amount of visual verification. ROOSTER has been successfully applied to targets observed by the Kepler main mission.
The pipeline is built around three random forest classifiers, each one in charge of a dedicated task: detecting rotational modulations, flagging close binary and classical pulsator candidates, and selecting the correct rotation period, respectively with the classifiers RotClass, PollFlag and PeriodSel.

We applied ROOSTER to the $\mathrm{K}$ and $\mathrm{M}$ main-sequence dwarfs analysed in S19 (Santos et al. 2019). We were able to detect rotation periods with an accuracy of $94.2 \%$ for a sample of 21707 stars (RotClass sample). Within the RotClass sample, we considered 14,562 stars with measured rotation periods (PeriodSel sample). The automatic analysis yields a true accuracy of $95.3 \%$ for PeriodSel, that is to say an agreement of the 
rotation period from ROOSTER within $10 \%$ of the reference value from S19. We noticed that the majority of the misclassified stars were due to known effects, in particular, instrumental modulations and confusion with a harmonic of the true rotation period. $31.6 \%$ of the stars of the PeriodSel sample are then flagged for visual checks which allows us to correct wrong values for 628 more stars and raises the PeriodSel accuracy to 99.5\%. Considering the results of the combined RotClass and PeriodSel steps on the PeriodSel sample, the global accuracy of ROOSTER is estimated at $92.1 \%$ without any visual check, and $96.9 \%$ after visual inspection of $25.2 \%$ of the RotClass sample (against 60\% in S19). By removing some input parameters from the ROOSTER training, we were also able to deal with stars with missing input parameters, at the price of a loss of accuracy. However, this accuracy loss is not only due to the diminution of the number of the parameters, but is also related to the intrinsic quality of the light curves for which the rotation pipeline was not able to provide a full set of parameters.

It should be emphasised that the accuracy estimated here is only valid for the K and M dwarf stars studied in S19. Rotation signals for hotter stars (mid $\mathrm{F}$ to $\mathrm{G}$ ) are more complex than for $\mathrm{K}$ and $\mathrm{M}$. In order to extend our analysis to those stars, a significant sample of hotter stars should first be introduced in the training set. The different accuracy values should also be reassessed before proceeding to the analysis. The length of the Kepler time series and the exquisite quality of the photometric measurements are important advantages for rotational analyses. To analyse light curves from K2 or TESS (which have a typical observational length significantly shorter than those of Kepler), first we need to verify whether the current training set is appropriate. If this is not the case, the classifiers must be trained with more suitable training sets (e.g by building a new training set with $\mathrm{K} 2$ or TESS stars). The selection of targets for visual inspection would probably be modified too.

Nevertheless, the global framework of ROOSTER can be adapted the Kepler F and $\mathrm{G}$ main-sequence solar type stars (Santos et al., in prep.). It should also be possible to use this technique to analyse data from K2, TESS and, in a near future, PLATO (PLAnetary Transit and Oscillations, Rauer et al. 2014). Such large-scale survey would be a great asset for gyrochronology models and our understanding of stellar spinning evolution in relation with age and spectral type.

Acknowledgements. We thank Suzanne Aigrain and Joe Llama for providing us with the simulated data used in Aigrain et al. (2015). S. N. B. L. B. and R. A. G. acknowledge the support from PLATO and GOLF CNES grants. A. R. G. S. acknowledges the support from NASA under grant NNX17AF27G. S. M. acknowledges the support from the Spanish Ministry of Science and Innovation with the Ramon y Cajal fellowship number RYC-2015-17697. P. L. P. and S. M. acknowledge support from the Spanish Ministry of Science and Innovation with the grant number PID2019-107187GB-I00. This research has made use of the NASA Exoplanet Archive, which is operated by the California Institute of Technology, under contract with the National Aeronautics and Space Administration under the Exoplanet Exploration Program. Software: Python (Van Rossum \& Drake 2009), numpy (Oliphant 2006), pandas (The pandas development team 2020; McKinney 2010), matplotlib (Hunter 2007), scikit-learn (Pedregosa et al. 2011). The source code used to obtain the present results can be found at: https://gitlab.com/sybreton/pushkin; https://gitlab.com/sybreton/ml_surface_rotation_paper.

\section{References}

Aerts, C., Mathis, S., \& Rogers, T. M. 2019, ARA\&A, 57, 35

Aigrain, S., Llama, J., Ceillier, T., et al. 2015, MNRAS, 450, 3211

Amazo-Gómez, E. M., Shapiro, A. I., Solanki, S. K., et al. 2020, A\&A, 636, A69

Angus, R., Aigrain, S., Foreman-Mackey, D., \& McQuillan, A. 2015, MNRAS, 450,1787
Angus, R., Morton, T., Aigrain, S., Foreman-Mackey, D., \& Rajpaul, V. 2018, MNRAS, 474, 2094

Angus, R., Beane, A., Price-Whelan, A. M., et al. 2020, AJ, 160, 90

Barnes, S. A. 2003, ApJ, 586, 464

Barnes, S. A. 2007, ApJ, 669, 1167

Benbakoura, M., Réville, V., Brun, A. S., Le Poncin-Lafitte, C., \& Mathis, S. 2019, A\&A, 621, A124

Berdyugina, S. V. 2005, Liv. Rev. Sol. Phys., 2, 8

Blancato, K., Ness, M., Huber, D., Lu, Y., \& Angus, R. 2020, ApJ, submitted [arXiv: 2005.09682]

Bolmont, E., \& Mathis, S. 2016, Celest. Mech. Dyn. Astron., 126, 275

Borucki, W. J., Koch, D., Basri, G., et al. 2010, Science, 327, 977

Breiman, L. 2001, Mach. Learn., 45, 5

Brown, T. M., Latham, D. W., Everett, M. E., \& Esquerdo, G. A. 2011, AJ, 142, 112

Brun, A. S., \& Browning, M. K. 2017, Liv. Rev. Sol. Phys., 14, 4 Bugnet, L., García, R. A., Davies, G. R., et al. 2018, A\&A, 620, A38 Bugnet, L., García, R. A., Mathur, S., et al. 2019, A\&A, 624, A79

Cameron, A. C. 2017, in The Impact of Stellar Activity on the Detection and

Characterization of Exoplanets, eds. H. J. Deeg, \& J. A. Belmonte (Cham: Springer International Publishing),

Ceillier, T., van Saders, J., García, R. A., et al. 2016, MNRAS, 456, 119

Ceillier, T., Tayar, J., Mathur, S., et al. 2017, A\&A, 605, A111

Domingo, V., Fleck, B., \& Poland, A. I. 1995, Sol. Phys., 162, 1

Eggenberger, P., Miglio, A., Montalban, J., et al. 2010, A\&A, 509, A72

Fröhlich, C., Romero, J., Roth, H., et al. 1995, Sol. Phys., 162, 101

García, R. A., \& Ballot, J. 2019, Liv. Rev. Sol. Phys., 16, 4

García, R. A., Hekker, S., Stello, D., et al. 2011, MNRAS, 414, L6

García, R. A., Ceillier, T., Salabert, D., et al. 2014a, A\&A, 572, A34

García, R. A., Mathur, S., Pires, S., et al. 2014b, A\&A, 568, A10

Howell, S. B., Sobeck, C., Haas, M., et al. 2014, PASP, 126, 398

Huber, D. 2018, in Asteroseismology and Exoplanets: Listening to the Stars and

Searching for New Worlds, eds. T. L. Campante, N. C. Santos, \& M. J. P. F. G. Monteiro, 49, 119

Huber, D., Bryson, S. T., Haas, M. R., et al. 2016, ApJS, 224, 2

Hunter, J. D. 2007, Comput. Sci. Eng., 9, 90

Jenkins, J. M., Caldwell, D. A., Chandrasekaran, H., et al. 2010, ApJ, 713, L87

Liu, Y., San Liang, X., \& Weisberg, R. H. 2007, J. Atmos. Oceanic Technol., 24, 2093

Lu, Y., Angus, R., Agüeros, M. A., et al. 2020, AJ, 160, 168

Mamajek, E. E., \& Hillenbrand, L. A. 2008, ApJ, 687, 1264

Mathis, S. 2015, A\&A, 580, L3

Mathur, S., García, R. A., Régulo, C., et al. 2010, A\&A, 511, A46

Mathur, S., García, R. A., Ballot, J., et al. 2014, A\&A, 562, A124

Mathur, S., Huber, D., Batalha, N. M., et al. 2017, ApJS, 229, 30

Mathur, S., García, R. A., Bugnet, L., et al. 2019, Front. Astron. Space Sci., 6, 46

McKinney, W. 2010, in Proceedings of the 9th Python in Science Conference, eds. S. van der Walt, \& J. Millman, 56

McQuillan, A., Mazeh, T., \& Aigrain, S. 2013, ApJ, 775, L11

McQuillan, A., Mazeh, T., \& Aigrain, S. 2014, ApJS, 211, 24

Meibom, S., Barnes, S. A., Latham, D. W., et al. 2011, ApJ, 733, L9

Meibom, S., Barnes, S. A., Platais, I., et al. 2015, Nature, 517, 589

Miglio, A., Chiappini, C., Morel, T., et al. 2013, MNRAS, 429, 423

Nielsen, M. B., Gizon, L., Schunker, H., \& Karoff, C. 2013, A\&A, 557, L10

Oliphant, T. 2006, NumPy: A guide to NumPy (USA: Trelgol Publishing)

The pandas development team 2020, https://github.com/pandas-dev/ pandas/

Pedregosa, F., Varoquaux, G., Gramfort, A., et al. 2011, J. Mach. Learn. Res., 12,2825

Pires, S., Mathur, S., García, R. A., et al. 2015, A\&A, 574, A18

Rauer, H., Catala, C., Aerts, C., et al. 2014, Exp. Astron., 38, 249

Reinhold, T., Reiners, A., \& Basri, G. 2013, A\&A, 560, A4

Ricker, G. R., Winn, J. N., Vanderspek, R., et al. 2015, J. Astron. Telesc. Instrum. Syst., 1, 014003

Santos, A. R. G., García, R. A., Mathur, S., et al. 2019, ApJS, 244, 21

Shapiro, A. I., Amazo-Gómez, E. M., Krivova, N. A., \& Solanki, S. K. 2020, A\&A, 633, A32

Simonian, G. V. A., Pinsonneault, M. H., \& Terndrup, D. M. 2019, ApJ, 871, 174

Skumanich, A. 1972, ApJ, 171, 565

Strassmeier, K. G. 2009, A\&ARv, 17, 251

Strugarek, A., Bolmont, E., Mathis, S., et al. 2017, ApJ, 847, L16

Torrence, C., \& Compo, G. P. 1998, Bull. Am. Meteorol. Soc., 79, 61

Van Rossum, G., \& Drake, F. L. 2009, Python 3 Reference Manual (Scotts Valley, CA: CreateSpace)

van Saders, J. L., Ceillier, T., Metcalfe, T. S., et al. 2016, Nature, 529, 181

Zhang, M., \& Penev, K. 2014, ApJ, 787, 131 


\section{Appendix A: Detailed parameter extraction methodology}

This appendix presents in detail of the methodology used to extract the $P_{\text {rot }}$ candidates considered by ROOSTER.

The first method used to measure $P_{\text {rot }}$ is a time-period analysis using a wavelet decomposition (Torrence \& Compo 1998; Liu et al. 2007; Mathur et al. 2010). Wavelets of different periods, each one taken as the convolution between a sinusoidal and a Gaussian function (Morlet wavelet), are cross-correlated with the light curve to obtain the wavelet power spectrum (WPS, see left panel of (b) in Fig. 2). The WPS is projected over the periodaxis to obtain the one-dimension Global Wavelet Power Spectrum (GWPS, see right panel of (b) in Fig. 2). Multiple Gaussian functions are fitted to the GWPS starting by the one with the highest amplitude. The fitted Gaussian function is removed and the next highest Gaussian peak is fitted in an iterative way until no peaks are above the noise level. Thus, the first period estimate, corresponding to the period of the highest fitted peak in the GWPS, is assigned as the rotation period recovered by this methodology: $P_{\text {GWPS }}$. The period uncertainty is taken as the half width at half maximum (HWHM) of the Gaussian function. Although this is a very conservative approach, it allows us to take into account variations due to differential rotation as part of the uncertainty.

The second analysis method is the ACF of the light curve. The rotation period, $P_{\mathrm{ACF}}$, corresponds to the period of the highest peak in the ACF at a lag greater than zero. Two other parameters are computed: $G_{\mathrm{ACF}}$ and $H_{\mathrm{ACF}}$. $G_{\mathrm{ACF}}$ is the height of $P_{\mathrm{ACF}}$, while $H_{\mathrm{ACF}}$ is the mean difference between the height of $P_{\mathrm{ACF}}$ and the two local minima on both sides of $P_{\mathrm{ACF}}$ (see an extended description in Ceillier et al. 2017).

The third method is the composite spectrum (CS). CS is the product between the normalized GWPS and ACF. This way, the peaks present in both GWPS and ACF (possibly related to rotation) are amplified while the signals appearing only in one of the two methods (for example due to instrumental effects that have a different manifestation in each analysis) are attenuated. Multiple Gaussian functions are fitted to the CS following the same iterative procedure as the one described for the GWPS. $P_{\mathrm{CS}}$ is obtained as the period of the fitted Gaussian of highest amplitude and the uncertainty is its HWHM. The amplitude of this peak is $H_{\mathrm{CS}}$.

Having three period estimates for each light curve $\left(P_{\mathrm{GWPS}}\right.$, $P_{\mathrm{ACF}}$, and $\left.P_{\mathrm{CS}}\right), \mathrm{S} 19$ computed the respective value for the photometric activity index $S_{\mathrm{ph}}$ (that is to say, one for each $P_{\text {rot }}$ estimation). $S_{\mathrm{ph}}$ is computed as defined by Mathur et al. (2014), being the standard deviation over light curve segments of $5 \times P_{\text {rot }}$. The final $S_{\text {ph }}$ value we provide corresponds to the average $S_{\mathrm{ph}} . S_{\mathrm{ph}}$ is corrected for the photon-shot noise following Jenkins et al. (2010). For some targets this correction leads to negative $S_{\mathrm{ph}}$ values. Most of the targets in this situation do not show rotational modulation. For those with rotational modulation and $S_{\mathrm{ph}}<0$ (a few percent of the targets), S19 applied individually a different correction to the photon-shot noise, which is computed from the high-frequency noise component in the power density spectrum. We note that the $S_{\mathrm{ph}}$ value has only a physical sense when rotational modulation is detected in the light curve and, thus, $P_{\text {rot }}$ is measured (e.g., Mathur et al. 2014, Egeland et al., in prep.).

In S19, a first group of stars with reliable rotation periods is automatically selected when there is a good agreement between the different period estimates and the heights for $P_{\mathrm{ACF}}$ and $P_{\mathrm{CS}}$ are larger than a given threshold (see S19 for details; height thresholds adopted from Ceillier et al. 2017). For the remainder of the targets (about 60\%), in order to decide whether the signal is related to rotational modulation and to select the correct $P_{\text {rot }}$, S19 visually inspected the respective light curves, rotation diagnostics, and power spectra. During the visual inspection $40 \%$ of the final selected $P_{\text {rot }}$ were recovered. There are different problems causing the measurement of a different $P_{\text {rot }}$ in each methodology. For example: small amplitudes of the rotational modulation, which translate into small values of $S_{\mathrm{ph}}, H_{\mathrm{ACF}}, G_{\mathrm{ACF}}$, and $H_{\mathrm{CS}}$; presence of instrumental modulations; and strong harmonic of $P_{\text {rot }}$. Instrumental modulations affect primarily the light curves obtained with the 55-day and 80-day filters. However, we note that depending on the $P_{\text {rot }}$ value, the correct period may not be recovered from the 20-day filter. Half of the rotation period can be wrongly retrieved as $P_{\text {rot }}$ (what we call strong harmonic) when the dominant spots producing the rotational signal are apart by $\sim 180^{\circ}$ in longitude. In our methodology, the GWPS is the most sensitive to this issue, while the ACF is the least sensitive. The performance of the ACF in these cases is discussed in detail by McQuillan et al. (2013, 2014). In S19, the decision on the filter used for the final $P_{\text {rot }}$ relies on two criteria: preserving the instrinsic rotational signal with the $S_{\mathrm{ph}}$ value not affected by filtering, while minimizing the impact of possible instrumental effects which may not alter the period estimate but may affect $S_{\mathrm{ph}}$. Typically the 20-day filter is selected for $P_{\text {rot }} \leq 23$ days, the 55-day filter is selected for $23<$ days $P_{\text {rot }} \leq 60$ days, and the 80-day filter is selected for $P_{\text {rot }}>60$ days. For the rotation period estimate itself the priority is given to $P_{\mathrm{GWPS}}$. The main reason for this choice is the conservative uncertainty for $P_{\text {GWPS }}$.

\section{Appendix B: Input parameters}

The detailed set of 159 parameters used to train RotClass, PollFlag and PeriodSel is presented in Table B.1. GWPS_GAUSS_1_i_XX, GWPS_GAUSS_2_i_XX and GWPS_GAUSS_3_i_XX respectively correspond to the amplitude, the central period and the standard deviation of the $i^{\text {th }}$ Gaussian fitted in the GWPS with the XX-day filter. The same naming convention has been used for CS_GAUSS_1_i_XX, CS_GAUSS_2_i_XX and CS_GAUSS_3_i_XX with the CS. 
Table B.1. 159 input parameters used to train the RF classifiers.

\begin{tabular}{|c|c|c|}
\hline ACF_ER_SPH_20 & ACF_ER_SPH_55 & ACF_ER_SPH_80 \\
\hline BAD_Q_FLAG & CS_CHIQ_20 & CS_CHIQ_55 \\
\hline CS_CHIQ_80 & CS_GAUSS_1_1_20 & CS_GAUSS_1_1_55 \\
\hline CS_GAUSS_1_1_80 & CS_GAUSS_1_2_20 & CS_GAUSS_1_2_55 \\
\hline CS_GAUSS_1_2_80 & CS_GAUSS_1_3_20 & CS_GAUSS_1_3_55 \\
\hline CS_GAUSS_1_3_80 & CS_GAUSS_1_4_20 & CS_GAUSS_1_4_55 \\
\hline CS_GAUSS_1_4_80 & CS_GAUSS_1_5_20 & CS_GAUSS_1_5_55 \\
\hline CS_GAUSS_1_5_80 & CS_GAUSS_2_1_20 & CS_GAUSS_2_1_55 \\
\hline CS_GAUSS_2_1_80 & CS_GAUSS_2_2_20 & CS_GAUSS_2_2_55 \\
\hline CS_GAUSS_2_2_80 & CS_GAUSS_2_3_20 & CS_GAUSS_2_3_55 \\
\hline CS_GAUSS_2_3_80 & CS_GAUSS_2_4_20 & CS_GAUSS_2_4_55 \\
\hline CS_GAUSS_2_4_80 & CS_GAUSS_2_5_20 & CS_GAUSS_2_5_55 \\
\hline CS_GAUSS_2_5_80 & CS_GAUSS_3_1_20 & CS_GAUSS_3_1_55 \\
\hline CS_GAUSS_3_1_80 & CS_GAUSS_3_2_20 & CS_GAUSS_3_2_55 \\
\hline CS_GAUSS_3_2_80 & CS_GAUSS_3_3_20 & CS_GAUSS_3_3_55 \\
\hline CS_GAUSS_3_3_80 & CS_GAUSS_3_4_20 & CS_GAUSS_3_4_55 \\
\hline CS_GAUSS_3_4_80 & CS_GAUSS_3_5_20 & CS_GAUSS_3_5_55 \\
\hline CS_GAUSS_3_5_80 & CS_NOISE_20 & CS_NOISE_55 \\
\hline CS_NOISE_80 & CS_N_FIT_20 & CS_N_FIT_55 \\
\hline CS_N_FIT_80 & CS_SPH_ER_20 & CS_SPH_ER_55 \\
\hline CS_SPH_ER_80 & END_TIME & F_07 \\
\hline F_20 & F_50 & F_7 \\
\hline GWPS_CHIQ_20 & GWPS_CHIQ_55 & GWPS_CHIQ_80 \\
\hline GWPS_GAUSS_1_1_20 & GWPS_GAUSS_1_1_55 & GWPS_GAUSS_1_1_80 \\
\hline GWPS_GAUSS_1_2_20 & GWPS_GAUSS_1_2_55 & GWPS_GAUSS_1_2_80 \\
\hline GWPS_GAUSS_1_3_20 & GWPS_GAUSS_1_3_55 & GWPS_GAUSS_1_3_80 \\
\hline GWPS_GAUSS_1_4_20 & GWPS_GAUSS_1_4_55 & GWPS_GAUSS_1_4_80 \\
\hline GWPS_GAUSS_1_5_20 & GWPS_GAUSS_1_5_55 & GWPS_GAUSS_1_5_80 \\
\hline GWPS_GAUSS_2_1_20 & GWPS_GAUSS_2_1_55 & GWPS_GAUSS_2_1_80 \\
\hline GWPS_GAUSS_2_2_20 & GWPS_GAUSS_2_2_55 & GWPS_GAUSS_2_2_80 \\
\hline GWPS_GAUSS_2_3_20 & GWPS_GAUSS_2_3_55 & GWPS_GAUSS_2_3_80 \\
\hline GWPS_GAUSS_2_4_20 & GWPS_GAUSS_2_4_55 & GWPS_GAUSS_2_4_80 \\
\hline GWPS_GAUSS_2_5_20 & GWPS_GAUSS_2_5_55 & GWPS_GAUSS_2_5_80 \\
\hline GWPS_GAUSS_3_1_20 & GWPS_GAUSS_3_1_55 & GWPS_GAUSS_3_1_80 \\
\hline GWPS_GAUSS_3_2_20 & GWPS_GAUSS_3_2_55 & GWPS_GAUSS_3_2_80 \\
\hline GWPS_GAUSS_3_3_20 & GWPS_GAUSS_3_3_55 & GWPS_GAUSS_3_3_80 \\
\hline GWPS_GAUSS_3_4_20 & GWPS_GAUSS_3_4_55 & GWPS_GAUSS_3_4_80 \\
\hline GWPS_GAUSS_3_5_20 & GWPS_GAUSS_3_5_55 & GWPS_GAUSS_3_5_80 \\
\hline GWPS_NOISE_20 & GWPS_NOISE_55 & GWPS_NOISE_80 \\
\hline GWPS_N_FIT_20 & GWPS_N_FIT_55 & GWPS_N_FIT_80 \\
\hline GWPS_SPH_ER_20 & GWPS_SPH_ER_55 & GWPS_SPH_ER_80 \\
\hline G_ACF_20 & G_ACF_55 & G_ACF_80 \\
\hline H_ACF_20 & H_ACF_55 & H_ACF_80 \\
\hline H_CS_20 & H_CS_55 & H_CS_80 \\
\hline LENGTH & N_BAD_Q & Prot_ACF_20 \\
\hline Prot_ACF_55 & Prot_ACF_80 & Prot_CS_20 \\
\hline Prot_CS_55 & Prot_CS_80 & Prot_GWPS_20 \\
\hline Prot_GWPS_55 & Prot_GWPS_80 & START_TIME \\
\hline Sph_ACF_20 & Sph_ACF_55 & Sph_ACF_80 \\
\hline Sph_CS_20 & Sph_CS_55 & Sph_CS_80 \\
\hline Sph_GWPS_20 & Sph_GWPS_55 & Sph_GWPS_80 \\
\hline Teff & kepmag & $\log 9$ \\
\hline
\end{tabular}

\section{Appendix C: ROOSTER performance with simulated data}

Aigrain et al. (2015) performed a hare and hounds exercise with simulated data. Several teams participated to the exercise, each one with their own methodology. The working sample was constituted of 1000 simulated light curves and five 1000-day solar light curves obtained with the Variability of Solar Irradiance and Gravity Oscillations (VIRGO, Fröhlich et al. 1995) instrument on board the Solar and Heliospheric Observatory (SoHO, Domingo et al. 1995). Among the 1000 simulated light curves, noise from real Kepler observations was added to 750, while the other 250 were kept noise-free.

The ROOSTER training methodology presented in this paper was slightly modified to be applied on the simulated data. The training set was based on the same $\mathrm{K}$ and $\mathrm{M}$ stars from S19. However, five stars were removed from the training set because they were also used as noise sources for the simulated light curves in Aigrain et al. (2015). RotClass and PeriodSel were trained without the stellar global parameters $\left(T_{\text {eff }}\right.$ and $\left.\log g\right)$, and the FliPer values. Furthermore, we also abandon the Kp, bad quarter flags, number of bad quarters in the light curves, lengths, start and end date of the light curves. ROOSTER was applied blindly to mimic the real working situation and the outputs were compared to the correct rotation periods only at the end.

Table C. 1 compares the ROOSTER results to those obtained by the CEA team in Aigrain et al. (2015). In Aigrain et al. (2015), a previous version of our rotation pipeline (see Appendix A for details on the version used in the current study) was used. For both (noisy and noise-free data), the ROOSTER accuracy (83.2\% compared to $88 \%$ ) is slightly lower than previously. Nevertheless, ROOSTER provided good rotation periods (that is to say periods within $10 \%$ of the median of the observable periods $P_{\text {obs }}$ defined in Aigrain et al. 2015) for $73.9 \%$ of the noisy light curves and $80.4 \%$ of the noise-free light curves. These results represent an improvement in comparison with the results from any method used in the hare and hounds exercise. In 2015, the CEA team had the best scores, with respectively $68.6 \%$ of good periods provided for noisy light curves and $75.4 \%$ for noise-free light curves.

In the hare and hounds exercise (Aigrain et al. 2015), stars were flagged as $o k$ if the detected rotation period (also considering the uncertainty) was good or within the range of observable periods $\left[P_{\mathrm{obs}, \min }, P_{\mathrm{obs}, \max }\right]$. A non-ok rotation period is denoted bad. Following this approach, ROOSTER was able to provide ok rotation periods for $83 \%$ of the noisy light curves and for $90 \%$ of the noise-free ones. Indeed, ROOSTER performs better than any method in the hare and hounds exercise. The comparison between ROOSTER periods $P_{\text {rot,ML }}$ and the reference values $P_{\mathrm{obs}}$ is shown in Fig. C.1.

Concerning the solar light curves, ROOSTER detected four rotation periods among the five. Two of those rotation periods are within the 25-30 days range and are therefore considered as correct.

As a final exercise, we decided to flag the light curves for visual check, similarly to what is described in Sect. 6. Light curves with a mean classification ratio between 0.4 and 0.8 were flagged. Additionally, from the subsample for which ROOSTER provided a period, we flagged (for details, see in Sect. 6):

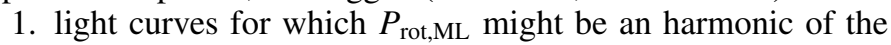
actual rotation period;

2. light curves with $P_{\text {rot,ML }}>38$ days or $P_{\text {rot,ML }}<1.6$ days;

3. light curves with a rotation period estimate not belonging to the proper filter.

This procedure led to 362 light curves being flagged among the Aigrain et al. (2015) sample of 1005 light curves. 41 of the noisy light curves and 9 of the noise-free light curves without detected period would have been proposed for visual inspection. Among the light curves with detected period, 36 noisy light curves and 7 noise-free light curves with bad period would have been visually checked. However, the visual check does not guarantee that the ROOSTER wrong determinations would be corrected. 
S. N. Breton et al.: ROOSTER: a machine-learning analysis tool for Kepler stellar rotation periods

Table C.1. Comparison between the 2015 CEA team results in the hare and hound exercise and the ROOSTER results from the simulated data.

\begin{tabular}{|c|c|c|c|c|c|c|c|c|c|c|c|c|}
\hline \multirow[t]{3}{*}{ Method } & \multicolumn{5}{|c|}{ Noisy } & \multicolumn{5}{|c|}{ Free } & \multicolumn{2}{|c|}{ Solar } \\
\hline & \multirow[t]{2}{*}{$\%$ det } & \multicolumn{2}{|c|}{$\%$ good } & \multicolumn{2}{|c|}{$\%$ ok } & \multirow[t]{2}{*}{$\%$ det } & \multicolumn{2}{|c|}{$\%$ good } & \multicolumn{2}{|c|}{$\%$ ok } & \multirow[t]{2}{*}{ No. det } & \multirow[t]{2}{*}{ No. ok } \\
\hline & & det & global & det & global & & det & global & det & global & & \\
\hline CEA 2015 & 78 & 88 & 68.6 & 95 & 74.1 & 82 & 92 & 75.4 & 99 & 81.2 & 2 & 2 \\
\hline ROOSTER & 88.8 & 83.2 & 73.9 & 93.5 & 83 & 92.8 & 86.6 & 80.4 & 97 & 90 & 4 & 2 \\
\hline
\end{tabular}

Notes. For each sample (noisy, noise-free and solar), percentage of detected (det) rotation periods is given, followed by percentage of good rotation periods among the detected rotation period and the full sample. The same percentages are also provided for $o k$ rotation periods. The good and $o k$ nomenclature in described in detail in Aigrain et al. (2015) as well as in Appendix C.

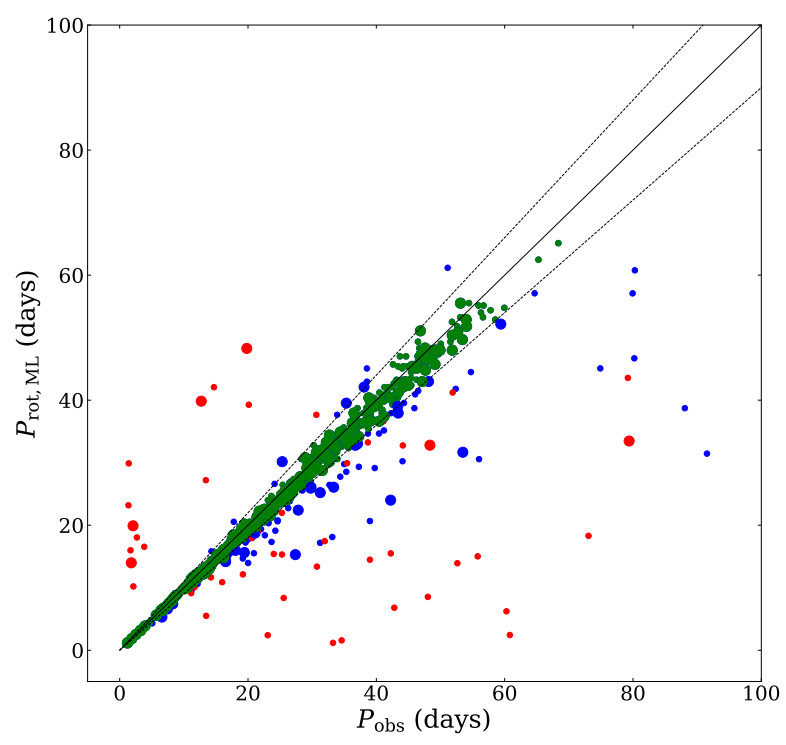

Fig. C.1. ROOSTER retrieved periods $P_{\text {rot,ML }}$ versus $P_{\text {obs }}$ from Aigrain et al. (2015). Good periods are shown in green, ok in blue, and bad in red. Large circles mark noise-free light curves, while small circles mark noisy light curves.

\section{Appendix D: Output format and visual check-flags signification}

The standard ROOSTER output files are saved as commaseparated values (csv) files. The column order of the standard ROOSTER output files is given in Table D.1, while the signification of the visual checks flag is summarised in Table D.2. The flags follow a hierarchical order, that is to say, if there is an
Table D.1. Column order of the output ROOSTER files.

\begin{tabular}{lc}
\hline \hline KIC & - \\
label RotClass & - \\
$P_{\text {rot,ML }}$ & days \\
$P_{\text {rot,error }}$ & days \\
$S_{\mathrm{ph}}$ & - \\
$S_{\mathrm{ph}, \mathrm{error}}$ & - \\
flag for visual check & (see Table D.2) \\
label FlagPoll & - \\
classification ratio Rot & - \\
classification ratio FlagPoll & - \\
flag missing parameters & - \\
$T_{\text {eff }}$ & $\mathrm{K}$ \\
$\log g$ & $\mathrm{dex}$ \\
\hline
\end{tabular}

Table D.2. Meaning of visual-check flags in ROOSTER output files.

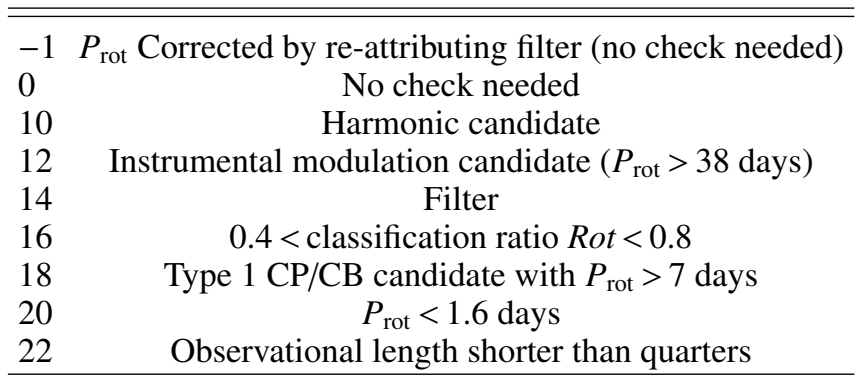

overlap of flags, the table will provide solely the first flag to be considered. 